\title{
DIAMETER MEASUREMENTS OF ASTEROIDS
}

\author{
AUDOUIN DOLLFUS \\ Observatoire de Paris
}

Direct optical measurements of asteroid diameters obtained by telescopic observations are scarce. Although several adequate instrumentations and techniques are available for the purpose, they have not been used. The importance of these determinations should be stressed for the attention of observers.

The filar micrometer was used only by one observer during the last century, and no additional measures have been made since. This was in 1894 and 1895 when E. Barnard (1902) used the $90 \mathrm{~cm}$ refractor of Lick Observatory, and the $100 \mathrm{~cm}$ refractor of Yerkes Observatory. The results are as follows:

Ceres: apparent diameter at $1 \mathrm{AU}: 1.060$, or $770 \mathrm{~km}$ (28 nights)

Pallas: apparent diameter at $1 \mathrm{AU}: 0.675$, or $490 \mathrm{~km}$ (5 nights)

Juno: apparent diameter at $1 \mathrm{AU}: 0.266$, or $195 \mathrm{~km}$ (5 nights)

Vesta: apparent diameter at $1 \mathrm{AU}: 0.531$, or $390 \mathrm{~km}$ (21 nights)

These filar micrometer measurements are difficult to make when the disks are only slightly larger than the image of the diffraction pattern blurred by atmospheric seeing; the accuracy is necessarily poor, especially for Juno; the last decimals given are not significant.

The interferometer with a double slit in the wavefront was used by M. Hamy (1899) with the $60 \mathrm{~cm}$ coude refractor of the Paris Observatory, but only on Vesta: apparent diameter at $1 \mathrm{AU}: 0.54$, or $400 \mathrm{~km}$ (8 nights).

Once again, this technique was not used again, despite the improvements in interferometric techniques and the larger telescopes now available.

The double-image micrometer has been used more recently in France by several collaborating observers. For descriptions of the double-image micrometer, see papers by P. Muller (1949) and A. Dollfus (1954). Although the survey is not yet complete, some of the as yet unpublished preliminary results are summarized in table I.

For small apparent diameters of only two to three times the effective resolving power of the telescope, large uncertainties remain in the measurements, and systematic errors occur. Some of them were computed or simulated at the laboratory by H. Camichel (1958), A. Dollfus (1963), and M. Hugon et al. (Camichel et al., 1964). 


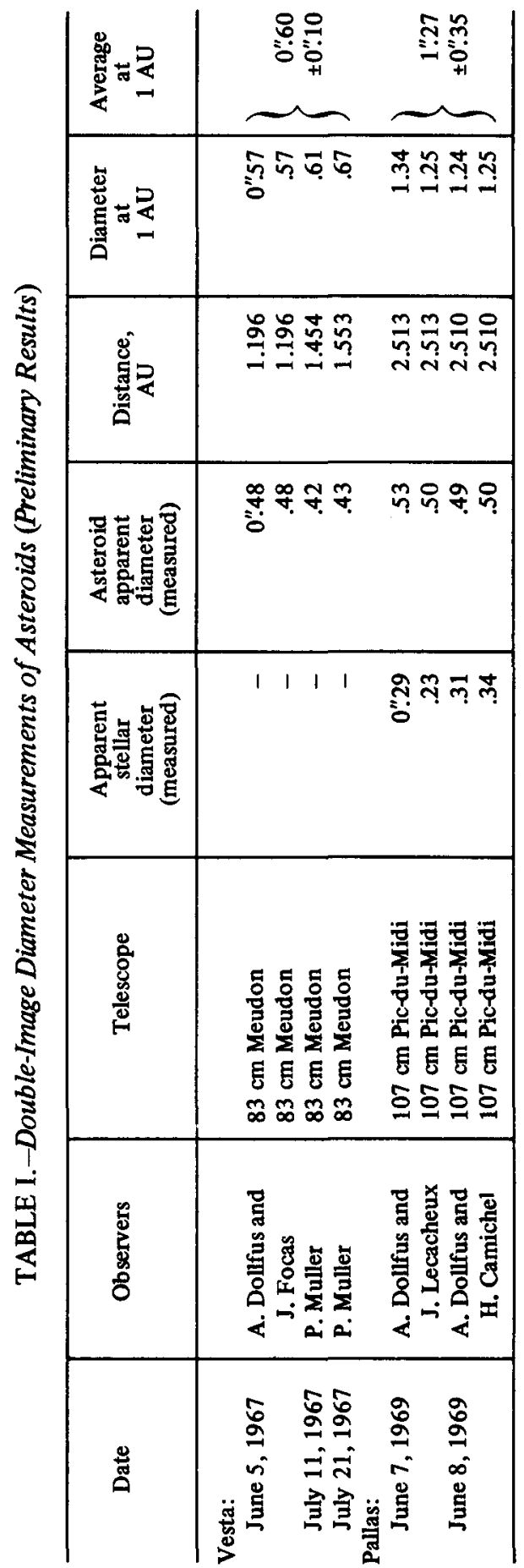


The error limits given in the table, \pm 0 ". 10 for Vesta and \pm 0 ". 35 for Pallas, are only estimates based on the practice of the laboratory simulations, taking into account the seeing conditions at the telescope when measurements were made.

The measurement of Vesta agrees with the values of Barnard (1902) and Hamy (1899); but the diameter of Pallas is larger than the Barnard value by a factor on the order of 2 . This very large discrepancy casts a doubt on the overall accuracy of the presently available determinations of asteroid diameters.

The diskmeter, designed by H. Camichel (1953), is a device producing a small artificial bright disk in the field of the telescope, with adjustable brightness, color, blurring, and diameter. Looking first at a nearby star, the observer adapts the brightness, color, and instrumental blurring for an artificial image of negligible apparent diameter, to reproduce as closely as possible the brightness configuration of the stellar image. Then, looking at the asteroid (or a small satellite), the observer readapts brightness and color, and without changing the blurring adjustment, increases the diameter of the artificial image until reproducing the behavior of the object in the field.

This kind of instrument was successfully used by $\mathrm{H}$. Camichel with the French Pic-du-Midi $60 \mathrm{~cm}$ refractor and by G.P. Kuiper with the Palomar $500 \mathrm{~cm}$ reflector on Neptune, Pluto, and planetary satellites. Kuiper used a diskmeter to measure some asteroids, but the details of this work have not been published. ${ }^{1}$

This technique seems to be particularly well adapted for asteroid diameter determinations and should be used.

The occultation of asteroids by the edge of the Moon provides curves of brightness variations with time, from which the apparent disk diameter can be derived with an excellent accuracy. For stellar objects of negligible apparent diameter, the photoelectric lightcurves recorded with a time resolution of a millisecond display a drop of brightness lasting some tenths of milliseconds, associated with at least one maximum and one minimum due to the diffraction pattern (see fig. 1); the higher order diffraction variations vanish into the noise

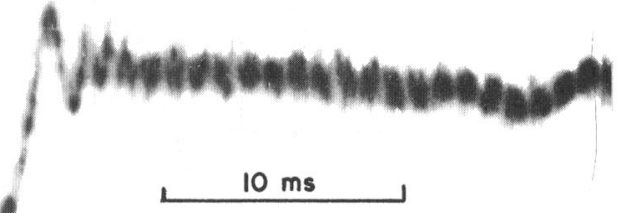

Figure 1.-Lightcurve of the lunar occultation of $\epsilon$-Capricorni, $4.7 \mathrm{mag}, \mathrm{B} 5 \mathrm{p}$. December 9 , 1964 , at $19^{\mathrm{h}} 31^{\mathrm{m}} 48^{\mathrm{s}}$; green filter. (60 cm telescope of Meudon Observatory, France; photoelectric observation by G. Spaak.)

${ }^{1}$ Note added in proof, by $T$. Gehrels: A summary paragraph occurs on p. 352 of Surfaces and Interiors of Planets and Satellites (ed., A. Dollfus; Academic Press, Inc.; London; 1970); the wording of that paragraph was checked with G. P. Kuiper. 


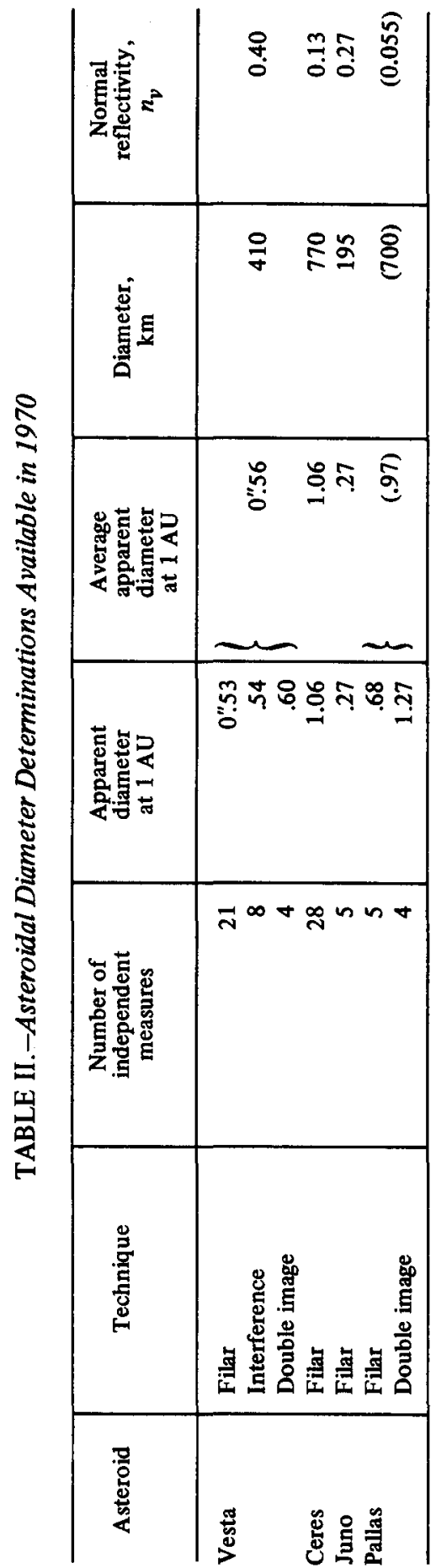


level. For some bright stars, small apparent diameters of the order of 0".005 are large enough to smear out the first minimum of light. Larger apparent diameters will change the slope of the lightcurve.

Figure 1 shows one of our curves obtained at Meudon Observatory on the star $\epsilon$-Capricorni, $4.7 \mathrm{mag}$, with a telescope of $60 \mathrm{~cm}$ diameter. A telescope of $200 \mathrm{~cm}$ produces the same noise level with a star of 7.3 mag. The brightest asteroids are expected to have apparent diameters larger than 0.05 , thus permitting a relaxation of the time resolution on the order of 30 times; the same noise level is obtained with a gain of brightness of $3.8 \mathrm{mag}$, and the technique reaches a magnitude of about 11 , provided by at least 15 asteroids. Further decrease of time resolution could be tried and larger telescopes used.

This interesting technique has not yet been used for asteroids.

\section{CONCLUSION}

The asteroidal diameter determinations currently available are summarized in table II.

The double-image micrometer, the interferometer, the diskmeter, and the lunar occultation photometry are suitable techniques available for refinement of these determinations and for their extension to a larger number of objects.

\section{REFERENCES}

Barnard, E. 1902, On the Dimensions of the Planets and Satellites. Astron. Nachr. 157, 260-268.

Camichel, H. 1953, Nouvelle Méthode de Mesure des Diamètres des Petits Astres et ses Résultats. Ann. Astrophys. 16, 41.

Camichel, H. 1958, Erreur Systématique sur la Mesure des Diamètres des Petits Astres Avec le Micrométre a Double Image. Ann. Astrophys. 21, 217-228.

Camichel, H., Hugon, M., and Rösch, J. 1964, Mesure du Diamètre de Mercure par la Méthode de Hertzsprung le 7 Novembre 1960. Icarus 3, 410-422.

Dollfus, A. 1954, L'Observation à la Tour Eiffel du Passage de Mercure Devant le Soleil Pour la Mesure de son Diametre. L'Astronomie 68, 337-345.

Dollfus, A. 1963, Mesure du Diametre de Mercure lors de son Passage Devant le Soleil le 7 Novembre 1960. Icarus 2, 219-225.

Hamy, M. 1899, Sur la Mesure Interférentielle des Petits Diamètres. Application aux Satellites de Jupiter et à Vesta. Bull. Astron. 16, 257-274.

Muller, P. 1949, Sur un Nouveau Micrométre à Double Image, ses Possibilités et Quelques Questions Connexes. Bull. Astron. 14, 177-313.

\section{DISCUSSION}

VEVERKA: You quoted the diameter measurements of the first four asteroids made by Barnard with a filar micrometer. The apparent diameter of Juno was given as 0 ". 3 . How meaningful, in your opinion, are such measurements when even at best "seeing" causes a smearing of 0.2 to 0.3 ? I realize that Barnard's measurements are internally quite consistent, but I would like to have an estimate of the absolute uncertainty involved, including systematic errors.

DOLLFUS: One of the purposes of my presentation was precisely to warn about the difficulty of the method. Bamard was a very experienced observer for filar micrometry, 
and was well aware of all the difficulties of the problem. However, we should not overestimate the accuracy of his measurements, and systematic error of 0.10 could be considered as highly probable. This is why the Barnard results, despite the high training and reputation of the author, should be checked when possible by other techniques.

GEHRELS: This does raise a problem. People like Barnard who did this type of work must have had a feeling of their precision and if I take it strictly from what you are saying then it would also concern Barnard's measurements of Ceres and Vesta. Should we not be allowed some confidence in these measures?

DOLLFUS: He did observations during several long periods of time but the gist lies in the systematic errors and against them accumulation of data does not really improve the result. The point is that the measures are distorted; one can in some respects simulate the conditions at the laboratory and study the systematic errors.

GEHRELS: In these measurements can you make a comment on which way the error might go? Should we expect measurements that are too large or too small?

DOLLFUS: Uncorrected filar measurements are too large; at the limit, a point source gives the apparent diameter of the diffraction pattern blurred by atmospheric turbulence.

KUIPER: Certainly Barnard should be considered a very keen observer. He was aware of what the atmosphere did to spoil the image and he would not use a night if it was not good. I have confidence in his diameters; the absolu te errors would be somewhat similar to the case of close binaries, and guessed to be 10 percent or perhaps from 20 to 25 percent, but they are real measures.

DOLLFUS: I was not clear in my statement. For disks with diameters of the order of the size of the diffraction pattern, a correction is to be applied. This is precisely the case of the asteroids; but it is difficult to judge what is the contribution of the diffraction if one includes the blurring due to atmospheric turbulence.

KUIPER: The diffraction was $0 " .12$ and the overall blurring not more than $0 " 2$. What counts is the square root of the sum of the squares, and therefore the diffraction is the least important in the final value. Certainly it has all the uncertainties that we are all aware of and Barnard was aware of. Values of the order of 0.5 would be essentially correct. Values smaller than 0.3 I would consider dubious.

CHAPMAN: There has been one recent test of the methods that have been used and described by Dollfus and Kuiper for determining asteroid diameters. Dollfus has summarized the modern attempts that have been made to determine the diameter of Neptune using these same methods. (See Dollfus, 1970.) All attempts to measure the diameter during the last half century prior to the recent stellar occultation by Neptune agreed with each other but differed from the correct value by more than 10 percent. The 2".5 diameter of Neptune is an order of magnitude larger than the largest asteroid diameters. Considering, in addition, that smearing by diffraction and seeing is of the same order as asteroid diameters, the failure of these methods to reach higher accuracy than 10 percent on Neptune (even taking account of the problems of limb darkening) casts doubt on any direct diameter measurements of asteroids.

DOLLFUS: The problem of the diameter determinations of Neptune is of a different nature: The limitations in Neptune measurements are altogether the lack of brightness and the limb darkening. Most of the earlier diameter measurements of Neptune concluded too small diameters because of the limb-darkening effect on a disk of very low brightness. However, our last telescopic measurements were obtained with the new Pic-du-Midi telescope, which gives three times more brightness than the previous one; a value of 2".23 was obtained, almost 10 percent larger than the previous measurements. This last value was considered by the observers as far better than the older. The value was later compared with the star occultation result; the agreement is within 3 percent and unexpectedly accurate in view of the difficulty of measuring so dark an object.

The case of minor planets is not the same. Observations are no longer limited by the lack of brightness; the average surface brightness is at least 30 times larger, with no limb 
darkening; the measurements, in this respect, are far easier. However, the diameters being smaller, careful laboratory analysis of the systematic errors introduced by the spreading of the images should be carried out for final evaluation.

\section{DISCUSSION REFERENCE}

Dollfus, A. 1970, Diamètres des Planètes et Satellites. Surfaces and Interiors of Planets and Satellites, ch. 2, pp. 45-139. Academic Press, Inc. London and New York. 